\title{
Hermitian harmonic maps from complete Hermitian manifolds to complete Riemannian manifolds
}

\author{
Lei $\mathbf{N i}^{\star}$ \\ Department of Mathematics, Purdue University, West Lafayette, IN 47907, USA \\ (e-mail: lni@math.purdue.edu)
}

Received June 25, 1997; in final form May 19, 1998

\section{Introduction}

In this paper we study a nonlinear elliptic system of equations imposed on a map from a complete Hermitian (non-Kähler) manifold to a Riemannian manifold. This system is more appropriate to Hermitian geometry than the harmonic map system since it is compatible with the holomorphic structure of the domain manifold in the sense that holomorphic maps are Hermitian harmonic maps. It was first studied by Jost and Yau in [J-Y], and was applied to study the rigidity of compact Hermitian manifolds. We extend their existence and uniqueness results to the case where both domain and target manifolds are complete. Hopefully the results will be useful to study corresponding rigidity of complete Hermitian manifolds.

Let $M$ be a complex manifold with Hermitian metric $\left(h_{\alpha \bar{\beta}}\right)$, and let $N$ be a Riemannian manifold with metric $\left(g_{i j}\right)$ and Christoffel symbols $\Gamma_{j k}^{i}$. A Hermitian harmonic map $u: M \rightarrow N$ satisfies the following elliptic system

$$
h^{\alpha \bar{\beta}}\left(\frac{\partial^{2} u^{i}}{\partial z^{\alpha} \partial \bar{z}^{\beta}}+\Gamma_{j k}^{i} \frac{\partial u^{j}}{\partial z^{\alpha}} \frac{\partial u^{k}}{\partial \bar{z}^{\beta}}\right)=0 .
$$

The related heat equation is

$$
\frac{\partial u^{i}}{\partial t}=h^{\alpha \bar{\beta}}\left(\frac{\partial^{2} u^{i}}{\partial z^{\alpha} \partial \bar{z}^{\beta}}+\Gamma_{j k}^{i} \frac{\partial u^{j}}{\partial z^{\alpha}} \frac{\partial u^{k}}{\partial \bar{z}^{\beta}}\right) .
$$

As it was pointed out in [J-Y] we know (1.1) is just the usual harmonic map equation if $M$ is Kählerian. Geometrically if we define the tension field by

\footnotetext{
* Research partially supported by NSF grant \#DMS-9626310
} 
$\sigma(u)=\nabla_{e_{A}}(d u)\left(e_{A}\right)$, and we use a holomorphic torsion-free connection on $\mathrm{M}$ instead of the Levi-Civita connection, then $\sigma(u) \equiv 0$ gives the equation (1.1). Since (1.1) has neither a divergence nor a variational structure it is harder to solve than the usual harmonic map system. One example in [J-Y] shows that even when $N$ is nonpositively curved the existence of Hermitian harmonic map in each homotopy class is not always guaranteed. However they proved the following solubility of the Dirichlet problem under the assumption that $N$ is nonpositively curved.

Theorem 6 [J-Y] (Jost-Yau). Let $M$ be a compact manifold with smooth boundary $\partial M$ and Hermitian metric $\left(h_{\alpha \bar{\beta}}\right)$, and let $N$ be a complete Riemannian manifold with nonpositive sectional curvature. Let $h: M \rightarrow N$ be a continuous map. Then there exists a unique Hermitian harmonic map $u: M \rightarrow N$ such that $\left.u\right|_{\partial M}=\left.h\right|_{\partial M}$ and $u$ is homotopic to $h$ w.r.t. the fixed boundary value.

We will use their result to prove the existence of Hermitian harmonic map from complete Hermitian manifolds to complete Riemannian manifolds by a compact exhaustion procedure under the assumption that the holomorphic Laplacian has positive first eigenvalue. This result can be thought as an analogy of the existence result for harmonic maps of Ding[D] and Li-Tam[LT]. The key point is to get a uniform energy density estimate. Here we derive the energy density estimate through the homotopy distance integral estimate, the mean value type inequality and a differential inequality for the homotopy distance functions. Even for the harmonic maps our method provides a different, perhaps simpler, proof of the previous existence results of Li-Tam and Ding. We also treat the parabolic equation in our paper. More precisely we first prove the existence of the global solution for the heat equation under the assumption that the holomorphic Laplacian has nonnegative first eigenvalue. Then we discuss when the solution of the heat equation converges to a Hermitian harmonic map.

The author would like to thank Prof. Peter Li for his generous support and many helpful discussions. The author would also like to thank the referee for many valuable comments. The thoroughness of the referee report were great help in presenting the paper in a readable form.

\section{Hermitian harmonic maps}

In this section we first sketch the definition of the Hermitian harmonic maps. Then we briefly state some basic properties of the Hermitian harmonic map and point out the difference and relation to usual harmonic maps and holomorphic maps.

Let $M$ be a Hermitian manifold of complex dimension $m$. Let $\left\{e_{A}\right\}, A=$ $1, \cdots, 2 m$ be a local orthonormal frame(real) of $M$. If $J$ is the almost com- 
plex structure we can always choose $e_{A}$ such that $\left\{e_{A}\right\}=\left\{e_{1}, e_{2}, \cdots, e_{m}\right.$, $\left.J e_{1}, J e_{2}, \cdots, J e_{m}\right\}$. The complex unitary frame will be $\left\{E_{\alpha}, \bar{E}_{\alpha}\right\}$ satisfying $e_{\alpha}=E_{\alpha}+\bar{E}_{\alpha}, J e_{\alpha}=\frac{1}{\sqrt{-1}}\left(\bar{E}_{\alpha}-E_{\alpha}\right)$ and that $\left\{E_{\alpha}\right\}$ spans the complex tangent spaces $T^{\prime}(M)$ locally.

Let $N$ be a Riemannian manifold of real dimension $n$, and let $u$ be a smooth map from $M$ to $N$. On $N$ we always choose the Levi-Civita connection which is compatible with the Riemannian structure. On $M$ now we choose connection such that it is compatible with the holomorphic structure on $M$ instead of the Riemannian structure. But for the convenience of the calculation we choose the connection to be torsion free. Of course the choice of the connection here is not unique and it is well-known that when $M$ is a Kähler manifold the Levi-Civita connection is both holomorphic and torsion free. In the following we denote the Levi-Civita connection by $\nabla$ and a chosen holomorphic torsion free connection by $\tilde{\nabla}$. Correspondingly we denote by $\Delta$ the standard Beltrami-Laplacian and by $\tilde{\Delta}$ the Laplacian of the holomorphic torsion free connection. Let $d u: T M \otimes C \rightarrow T N \otimes C$ to be the tangent map of $u$. Then one can define $\nabla d u(X, Y)$ by

$$
\nabla d u(X, Y)=\nabla_{Y} d u(X)-d u\left(\tilde{\nabla}_{X} Y\right) .
$$

The torsion free assumption makes the above defined $\nabla d u(\cdot, \cdot)$ symmetric.

It is natural to define the tension fields of the map $u$ to be the trace of $\nabla d u(\cdot, \cdot)$, namely

$$
\begin{aligned}
\sigma(u) & =\frac{1}{4} \sum_{A=1}^{2 m} \nabla d u\left(e_{A}, e_{A}\right) \\
& =\frac{1}{4}\left(\sum_{\alpha=1}^{m} \nabla d u\left(e_{\alpha}, e_{\alpha}\right)+\sum_{\alpha=1}^{m} \nabla d u\left(J e_{a}, J e_{a}\right)\right) .
\end{aligned}
$$

The factor $\frac{1}{4}$ here is used to normalize the notation such that the Laplacian operator of this connection for functions is given by

$$
\tilde{\Delta}=h^{\beta \bar{\gamma}} \frac{\partial^{2}}{\partial z^{\beta} \partial \bar{z}^{\gamma}}
$$

By definition we call a smooth map $u$ to be Hermitian harmonic if the above defined tension field $\sigma(u) \equiv 0$. A direct calculation as follows shows 
that in the complex local coordinates it is equivalent to the equation (1.1):

$$
\begin{aligned}
\sigma(u)= & \frac{1}{4}\left(\sum_{\alpha=1}^{m} \nabla d u\left(e_{\alpha}, e_{\alpha}\right)+\sum_{\alpha=1}^{m} \nabla d u\left(J e_{a}, J e_{a}\right)\right) \\
= & \frac{1}{4}\left(\sum_{\alpha=1}^{m} \nabla d u\left(E_{\alpha}+\bar{E}_{\alpha}, E_{\alpha}+\bar{E}_{\alpha}\right)\right. \\
& \left.+\sum_{\alpha=1}^{m} \nabla d u\left(\bar{E}_{\alpha}-E_{\alpha}, \bar{E}_{\alpha}-E_{\alpha}\right)\right) \\
= & \sum_{\alpha=1}^{m} \nabla d u\left(E_{\alpha}, \bar{E}_{\alpha}\right) \\
= & \sum^{\beta \bar{\gamma}} \nabla d u\left(\frac{\partial}{\partial z^{\beta}}, \frac{\partial}{\partial \bar{z}^{\gamma}}\right) \\
= & \left(\tilde{\Delta} u^{i}+\Gamma_{j k}^{i} \frac{\partial u^{j}}{\partial z^{\beta}} \frac{\partial u^{k}}{\partial \bar{z}^{\gamma}} h^{\beta \bar{\gamma}}\right) \frac{\partial}{\partial u^{i}} \\
& +\left(\tilde{\Delta} u^{i}+\Gamma_{j k}^{i} \frac{\partial u^{j}}{\partial z^{\beta}} \frac{\partial u^{k}}{\partial \bar{z}^{\gamma}} h^{\beta \bar{\gamma}}\right) \frac{\partial}{\partial \bar{u}^{i}} .
\end{aligned}
$$

If we denote the tension field for the usual harmonic map by $\tau(u)$, then the difference is given by

$$
(\sigma(u))^{i}-(\tau(u))^{i}=(\tilde{\Delta}-\Delta) u^{i} .
$$

The difference of the two Laplacians is given by a first order differential operator as follows

$$
(\tilde{\Delta}-\Delta) f=\left(\tilde{\nabla}_{e_{A}} e_{A}-\nabla_{e_{A}} e_{A}\right) f=\langle V, \nabla f\rangle,
$$

where $\tilde{\nabla}$ is the holomorphic connection and $V$ is a well-defined vector fields on $M$, which depends on the first derivatives of $h^{\alpha \bar{\beta}}$.

The basic properties of Hermitian harmonic maps contain

1) If $N$ is a Kähler manifold, a holomorphic map is a Hermitian harmonic map.

2) If $\varphi: M_{1} \rightarrow M_{2}$ is holomorphic and $u: M_{2} \rightarrow N$ is Hermitian harmonic, the composition $u \circ \varphi$ is a Hermitian harmonic map.

3) If $u: M \rightarrow N_{1}$ is a Hermitian harmonic map and $\varphi: N_{1} \rightarrow N_{2}$ is a totally geodesic immersion then $\varphi \circ u$ is Hermitian harmonic.

There might be other ways to define Hermitian harmonic maps which are compatible with the holomorphic structure. For example the most natural choice is the Hermitian holomorphic connection, which is widely used in the study of Hermitian holomorphic vector bundle and Hermitian geometry. Or 
we can use Hermitian holomorphic connections on both domain and target manifolds when they are both complex. The problem for this connection is that it is not torsion free when $M$ is not a Kähler manifold. This makes the analysis even harder since we no longer have nice differential inequalities. At last we should point out that even when our choice of connection on the domain manifold is not compatible with the metric, the Levi-Civita connection on the target manifold implies that the pull-back connection over the pull-back bundle $u^{*} T N$ is Riemannian. This fact is very important in our basic estimates in the next section.

\section{Basic differential inequalities}

Let $M$ be a complex manifold with Hermitian metric $\left(h_{\alpha \bar{\beta}}\right)$, and let $N$ be a Riemannian manifold with metric $\left(g_{i j}\right)$ and Christoffel symbols $\Gamma_{j k}^{i}$. We will always assume that $N$ has nonpositive sectional curvature. Let $u$ be a smooth map from $M$ to $N$. The energy density $e(u)$ of the map $u$ is defined as $\|d u\|^{2}$, or in local coordinates, $h^{\alpha \bar{\beta}} g_{i j}(u) \frac{\partial u^{i}}{\partial z^{\alpha}} \frac{\partial u^{j}}{\partial z^{\beta}}$. The first useful lemma is the following differential inequality on the energy density function proved by Jost-Yau [J-Y].

Lemma 3.1 (J-Y). Suppose that $N$ has nonpositive sectional curvature and $u$ is a Hermitian harmonic map from $M$ to $N$ then the energy density function e(u) satisfies

$$
\tilde{\Delta} e(u) \geq \frac{1}{2}\|\nabla d u\|^{2}-C e(u)
$$

where $C$ depends on the pointwise upper bound of both first and second derivatives of $h^{\alpha, \bar{\beta}}$.

Proposition 3.2. Let $N$ be a Riemannian manifold with nonpositive sectional curvature. If $u_{1}$ and $u_{2}$ are two smooth maps defined over some domain $\Omega$ to $N$, and $U(x, s): \Omega \times[0,1] \rightarrow N$ is the geodesic homotopy between them (It always exists by [S-Y]), then

$$
\int_{0}^{1}\left\|\nabla d U\left(\frac{\partial}{\partial s}\right)\right\|^{2} d s \geq \frac{1}{2}\left(e\left(u_{1}\right)-3 e\left(u_{2}\right)\right) .
$$

Proof. We first prove the following lemma.

Lemma 3.3. Let $e(s)$ be the energy density function of $U(x, s)$, where $U(x, s)$ is the geodesic homotopy between $u_{1}$ and $u_{2}$, then

$$
\left(\frac{d^{2}}{d s^{2}}\right) e(s) \geq 0
$$

i.e. $e(s)$ is a convex function of $s$. 
Proof of Lemma 3.3.

Let $e_{A}$ be the local orthonormal frame for $M$, then $e(s)=\left\|d U\left(e_{A}\right)\right\|^{2}$ by definition. Then we have the following calculation:

$$
\begin{aligned}
\frac{d}{d s} e(s) & =2\left\langle\nabla_{\frac{\partial}{\partial s}} d U\left(e_{A}\right), d U\left(e_{A}\right)\right\rangle \\
& =2\left\langle\nabla_{e_{A}} d U\left(\frac{\partial}{\partial s}\right), d U\left(e_{A}\right)\right\rangle,
\end{aligned}
$$

and

$$
\begin{aligned}
\frac{d^{2}}{d s^{2}} e(s)= & 2\left\langle\nabla_{e_{A}} d U\left(\frac{\partial}{\partial s}\right), \nabla_{e_{A}} d U\left(\frac{\partial}{\partial s}\right)\right\rangle \\
& +2\left\langle\nabla_{e_{A}}\left(\nabla_{\frac{\partial}{\partial s}} d U\left(\frac{\partial}{\partial s}\right)\right), d U\left(e_{A}\right)\right\rangle \\
- & 2\left\langle R^{N}\left(d U\left(\frac{\partial}{\partial s}\right), d U\left(e_{A}\right)\right) d U\left(\frac{\partial}{\partial s}\right), d U\left(e_{A}\right)\right\rangle \\
= & 2\left\langle\nabla_{e_{A}} d U\left(\frac{\partial}{\partial s}\right), \nabla_{e_{A}} d U\left(\frac{\partial}{\partial s}\right)\right\rangle \\
& -2\left\langle R^{N}\left(d U\left(\frac{\partial}{\partial s}\right), d U\left(e_{A}\right)\right) d U\left(\frac{\partial}{\partial s}\right), d U\left(e_{A}\right)\right\rangle \\
\geq & 0 .
\end{aligned}
$$

Here we have used the fact that the connection over domain manifold is torsion free and that the connection over target manifold is Riemannian as well as the fact that $U(s, \cdot)$ is a geodesic.

Remark 3.1. The convexity of the energy density might be an interpretation of the convexity of the distance functions on nonpositive curved manifolds.

Now we can begin to prove Proposition 3.2. From Lemma 3.3 we have

$$
\int_{0}^{1} e(s) d s \leq \frac{1}{2}\left(e\left(u_{1}\right)+e\left(u_{2}\right)\right) .
$$

On the other hand we have

$$
\begin{gathered}
e\left(u_{1}\right)-e\left(u_{2}\right)=e(1)-e(0) \\
=2 \int_{0}^{1}<\nabla_{\frac{\partial}{\partial s}} d U\left(e_{A}\right), d U\left(e_{A}\right)>d s \\
\leq \int_{0}^{1}\left\|\nabla d U\left(\frac{\partial}{\partial s}\right)\right\|^{2} d s+\int_{0}^{1}\left\|d U\left(e_{A}\right)\right\|^{2} d s \\
\leq \int_{0}^{1}\left\|\nabla d U\left(\frac{\partial}{\partial s}\right)\right\|^{2} d s+\frac{1}{2}\left(e\left(u_{1}\right)+e\left(u_{2}\right)\right) .
\end{gathered}
$$

Rewriting (3.4) we have the claim of Proposition 3.2. 
Lemma 3.4. Let $u$ and $v$ be two smooth maps from $M$ into $N$ with nonpositive sectional curvature and let $U(x, s)$ be the geodesic homotopy between them. If we denote by $\rho^{2}$ the square of the homotopy distance between $u$ and $v$, then we have

$$
\tilde{\Delta} \rho^{2} \geq 2 \int_{0}^{1}\left\|\nabla d U\left(\frac{\partial}{\partial s}\right)\right\|^{2} d s-2 \rho(\|\sigma(u)\|+\|\sigma(v)\|) .
$$

Proof. The proof is just direct calculation.

By definition

$$
\rho^{2}=\int_{0}^{1}\left\|d U\left(\frac{\partial}{\partial s}\right)\right\|^{2} d s .
$$

Therefore

$$
\begin{aligned}
\tilde{\Delta} \rho^{2}= & \left(e_{\alpha} e_{\alpha}-\bar{\nabla}_{e_{\alpha}} e_{\alpha}\right) \int_{0}^{1}\left\|d U\left(\frac{\partial}{\partial s}\right)\right\|^{2} d s \\
= & 2 \int_{0}^{1}\left\|\nabla_{e_{\alpha}} d U\left(\frac{\partial}{\partial s}\right)\right\|^{2} d s \\
& +2 \int_{0}^{1}\left\langle\nabla_{e_{\alpha}} \nabla_{\frac{\partial}{\partial s}} d U\left(e_{\alpha}\right), d U\left(\frac{\partial}{\partial s}\right)\right\rangle d s \\
& -2 \int_{0}^{1}\left\langle\nabla_{\bar{\nabla}_{e_{\alpha}} e_{\alpha}} d U\left(\frac{\partial}{\partial s}\right), d U\left(\frac{\partial}{\partial s}\right)\right\rangle d s \\
\geq & 2 \int_{0}^{1}\left\|\nabla_{e_{\alpha}} d U\left(\frac{\partial}{\partial s}\right)\right\|^{2} d s \\
& +2 \int_{0}^{1}\left\langle\nabla_{\frac{\partial}{\partial s}}\left(\nabla_{e_{\alpha}} d U\left(e_{\alpha}\right)-d U\left(\bar{\nabla}_{e_{\alpha}} e_{\alpha}\right)\right), d U\left(\frac{\partial}{\partial s}\right)\right\rangle d s \\
\geq & 2 \int_{0}^{1}\left\|\nabla_{e_{\alpha}} d U\left(\frac{\partial}{\partial s}\right)\right\|^{2} d s \\
& +\left.2\left\langle\left(\nabla_{e_{\alpha}} d U\left(e_{\alpha}\right)-d U\left(\bar{\nabla}_{e_{\alpha}} e_{\alpha}\right)\right), d U\left(\frac{\partial}{\partial s}\right)\right\rangle\right|_{0} ^{1} \\
\geq & 2 \int_{0}^{1}\left\|\nabla_{e_{\alpha}} d U\left(\frac{\partial}{\partial s}\right)\right\|^{2}-2 \rho(\|\sigma(u)\|+\|\sigma(v)\|) .
\end{aligned}
$$

As a corollary we have that if $u$ and $v$ are all Hermitian harmonic maps then

$$
\tilde{\Delta} \rho^{2} \geq 2 \int_{0}^{1}\left\|\nabla d U\left(\frac{\partial}{\partial s}\right)\right\|^{2} d s .
$$

Corollary 3.5. Let $M, N$ and $u, v$ as in Lemma 3.4. Then the following differential inequality holds;

$$
\tilde{\Delta} \rho \geq-(\|\sigma(u)\|+\|\sigma(v)\|) .
$$


Proof. The similar inequality for the usual harmonic maps was proved by Schoen-Yau in [S-Y].

By Lemma 3.4 and

$$
\begin{aligned}
\tilde{\Delta} \rho^{2} & =\Delta \rho^{2}+\left\langle V, \nabla \rho^{2}\right\rangle \\
& =2 \rho \Delta \rho+2 \rho\langle V, \rho\rangle+2|\nabla \rho|^{2} \\
& =2 \rho \tilde{\Delta} \rho+2|\nabla \rho|^{2},
\end{aligned}
$$

it will be sufficient to show that

$$
|\nabla \rho|^{2} \leq \int_{0}^{1}\left\|\nabla d U\left(\frac{\partial}{\partial s}\right)\right\|^{2} d s .
$$

This follows from

$$
\left|\nabla \rho^{2}\right| \leq 2 \rho\left(\int_{0}^{1}\left\|\nabla d U\left(\frac{\partial}{\partial s}\right)\right\|^{2} d s\right)^{\frac{1}{2}} .
$$

The inequality (3.8) can be proved as follows;

$$
\begin{aligned}
\left|\nabla \rho^{2}\right| & \leq\left|\int_{0}^{1} 2<d U\left(\frac{\partial}{\partial s}\right), \nabla d U\left(\frac{\partial}{\partial s}\right)>d s\right| \\
& \leq 2\left(\int_{0}^{1}\left\|d U\left(\frac{\partial}{\partial s}\right)\right\|^{2} d s\right)^{\frac{1}{2}}\left(\int_{0}^{1}\left\|\nabla d U\left(\frac{\partial}{\partial s}\right)\right\|^{2} d s\right)^{\frac{1}{2}} \\
& =2 \rho\left(\int_{0}^{1}\left\|\nabla d U\left(\frac{\partial}{\partial s}\right)\right\|^{2} d s\right)^{\frac{1}{2}} .
\end{aligned}
$$

Remark 3.2. A point-wise gradient estimate for harmonic maps was proved in $[\mathrm{Ch}]$ and corresponding estimate for heat equation was derived in [LJ]. In the theory of harmonic maps these gradient estimates play a very important role in both existence and Liouville type results. While one can see from the next section that in order to prove the existence result we only need a differential type inequality and some integral estimates due to the meanvalue type inequalities of Li-Schoen and Li-Tam (for the heat equation). On the other hand, we want to point out here that the Liouville type theorems for the usual harmonic maps can also be proved by this approach without using the point-wise gradient estimate. For example one can reprove Cheng's theorem by the following argument;

Let $u$ be a harmonic map from $M$ to $N$, where $M$ has nonnegative Ricci curvature and $N$ is simply connected and has nonpositive sectional curvature. Let $d\left(\cdot, y_{o}\right)$ be the distance function of $N$ to a fixed point $y_{o}$ and let $d(x)=d\left(u(x), y_{o}\right)$. The similar proof as of Proposition 3.1 shows that

$$
\Delta d^{2} \geq 2 e(u)
$$


Let $\varphi=\varphi(r(x))$ be a cut-off function on $M$, where $r(x)$ is distance function of $M$. Multiply $\varphi^{2}$ on both sides of the above inequality and integrate by parts we have

$$
\begin{aligned}
\int_{M} e(u) \varphi^{2} & \leq \int_{M} \Delta d^{2} \varphi^{2} \\
& =-4 \int_{M}\langle\nabla \varphi, \nabla d\rangle d \varphi \\
& \leq 4\left(\int_{M}|\nabla \varphi|^{2} d^{2}\right)^{\frac{1}{2}}\left(\int_{M}|\nabla d|^{2} \varphi^{2}\right)^{\frac{1}{2}} \\
& \leq 4\left(\int_{M}|\nabla \varphi|^{2} d^{2}\right)^{\frac{1}{2}}\left(\int_{M} e(u) \varphi^{2}\right)^{\frac{1}{2}} .
\end{aligned}
$$

Here we used the fact that $|\nabla d|^{2} \leq e(u)$. From the above we can easily see that

$$
\int_{M} e(u) \varphi^{2} \leq 16 \int_{M}|\nabla \varphi|^{2} d^{2},
$$

which implies that

$$
\int_{B(r)} e(u) \leq \frac{16}{r^{2}} \int_{B(2 r)} d^{2} .
$$

It is a well-known fact that $e(u)$ is subharmonic, therefore satisfies meanvalue inequality. Once we assume that $d(x)=o(r(x))$ one can easily see from the above inequality and the mean value inequality that $e(u) \equiv 0$ since

$$
\begin{aligned}
\sup _{B\left(\frac{r}{2}\right)} e(u) & \leq \frac{C(M)}{V(r)} \int_{B(r)} e(u) \\
& \leq \frac{C(M)\left(\sup _{B(2 r)} d^{2}\right) V(2 r)}{r^{2} V(r)} \rightarrow 0 .
\end{aligned}
$$

The advantage of this type argument is that not only it is simpler than the gradient estimate but also it can be modified to allow quasi-isometries of the domain manifold $M$. This point has been exploited by Peter Li and J. Wang in the study of the convex hull property of harmonic maps (cf. [L-W]).

\section{Existence of the Hermitian harmonic maps}

In this section we prove an existence theorem for Hermitian harmonic maps between complete manifolds. First we fix some notations. We say a second order elliptic operator $L$ has positive first eigenvalue $\lambda_{1}(L, M)$ if for any compact supported smooth function $\varphi$ one has 


$$
\int_{M}(-L \varphi) \varphi \geq \lambda_{1}(L, M) \int_{M} \varphi^{2}
$$

In particular, we simply denote $\lambda_{1}(L, M)$ by $\lambda_{1}(M)$ when $L$ is the BeltramiLaplacian operator and we denote $\lambda_{1}(L, M)$ by $\tilde{\lambda}_{1}(M)$ when $L$ is the holomorphic Laplacian operator $\tilde{\Delta}$ of the chosen holomorphic torsion free connection.

Theorem 4.1. Assume that $M$ is a complete Hermitian manifold with positive first eigenvalue $\tilde{\lambda}_{1}(M)$ for $\tilde{\Delta}, N$ is a complete Riemannian manifold with nonpositive sectional curvature and $h$ is a smooth map from $M$ to $N$. If there is a positive number $p \geq 1$ such that $h$ has bounded $\|\sigma(u)\|_{L^{2 p}}$ then there exists a Hermitian harmonic map $u: M \rightarrow N$ such that $u$ is homotopic to $h$.

Remark 4.1. For the usual harmonic maps the existence result of this type was proved by Ding [D] and Li-Tam [L-T] under the similar assumption as ours here. The argument here in fact also provides a simple proof for the usual harmonic maps if one replace Jost-Yau's theorem by using Hamilton's solution for the Dirichlet problem of harmonic maps.

We prove the result by the compact exhaustion procedure. Let $\left\{\Omega_{i}\right\}$ be a compact exhaustion of $M$ with, at least, Lipschitz boundary. By theorem 6 in $[\mathrm{J}-\mathrm{Y}]$ we have $\left\{u_{i}\right\}$ which solve the Dirichlet homotopy problem

$$
\left\{\begin{array}{l}
\sigma\left(u_{i}\right) \equiv 0 \\
\left.u_{i}\right|_{\partial \Omega_{i}}=h, u_{i} \sim h \text { rel } \partial \Omega_{i}
\end{array}\right.
$$

We will argue that there exists a subsequence of $\left\{u_{i}\right\}$ such that it converges to a Hermitian harmonic map on the whole manifold. In order to do that we use the estimate in Sect. 3 to bound the energy density over the compact domain $K$, or $B_{o}(R)$. The basic technique is to look at $\rho_{i}^{2}(x)$, the square of the homotopy distance between $u_{i}$ and $h$, and $\rho_{i j}^{2}(x)$, the square of the homotopy distance between $u_{i}$ and $u_{j}(i>j)$. In the following we first try to show that $\rho_{i}$ (therefore $\rho_{i j}$ ) has uniform $L^{2 p}$ norm.

By Corollary 3.5, after we replace $u$ by $u_{i}$, a Hermitian harmonic map and replace $v$ by the initial map $h$, we have

$$
\tilde{\Delta} \rho_{i} \geq-\|\sigma(h)\|
$$


Multiplying $\rho_{i}^{2 p-1}$ on the both sides of the above inequality and integrating by parts, we then have

$$
\begin{aligned}
-(2 p-1) \int_{M}\left|\nabla \rho_{i}\right|^{2} \rho_{i}^{2 p-2} & \\
+\int_{M}\left\langle V, \nabla \rho_{i}\right\rangle \rho_{i}^{2 p-1} & =\int_{M}\left(\tilde{\Delta} \rho_{i}\right) \rho_{i}^{2 p-1} \\
& \geq-\int_{M}\|\sigma(h)\| \rho_{i}^{2 p-1} .
\end{aligned}
$$

Here we have used the fact that $\rho_{i}=0$ on $\partial \Omega_{i}$ since $u_{i}$ coincides with $h$ on the boundary of $\Omega_{i}$. Multiplying $p$ on both sides we get

$$
\begin{aligned}
p \int_{M}\|\sigma(h)\| \rho_{i}^{2 p-1} \geq & (2 p-1) p \int_{M}\left|\nabla \rho_{i}\right|^{2} \rho_{i}^{2 p-2} \\
& -p \int_{M}\left\langle V, \nabla \rho_{i}\right\rangle \rho_{i}^{2 p-1} .
\end{aligned}
$$

On the other hand, by the assumption that $\tilde{\lambda}_{1}(M)>0$, we have that for any compact supported function $\varphi$

$$
\int_{M}(-\tilde{\Delta} \varphi) \varphi \geq \tilde{\lambda}_{1}(M) \int_{M} \varphi^{2},
$$

or

$$
\int_{M}|\nabla \varphi|^{2}-\int_{M}\langle V, \nabla \varphi\rangle \varphi \geq \tilde{\lambda}_{1}(M) \int_{M} \varphi^{2} .
$$

Letting $\varphi=\rho_{i}^{p}$, we get

$$
p^{2} \int_{M} \rho_{i}^{2 p-2}\left|\nabla \rho_{i}\right|^{2}-p \int_{M}<V, \nabla \rho_{i}>\rho_{i}^{2 p-1} \geq \tilde{\lambda}_{1}(M) \int_{M} \rho_{i}^{2 p}
$$

Adding (4.2) and (4.3) we have

$$
p \int_{M}\|\sigma(h)\| \rho_{i}^{2 p-1} \geq\left(p^{2}-p\right) \int_{M}\left|\nabla \rho_{i}\right|^{2} \rho_{i}^{2 p-2}+\tilde{\lambda}_{1}(M) \int_{M} \rho_{i}^{2 p} .
$$

In particular, for $p \geq 1$ we have

$$
p \int_{\Omega_{i}}\|\sigma(h)\| \rho_{i}^{2 p-1} \geq \tilde{\lambda}_{1}(M) \int_{\Omega_{i}} \rho_{i}^{2 p}
$$

Here we replace $M$ by $\Omega_{i}$ since $\rho_{i}$ is compact-supported in $\Omega_{i}$. Hölder inequality and (4.5) together imply that

$$
p\left(\int_{\Omega_{i}} \rho_{i}^{2 p}\right)^{\frac{2 p-1}{2 p}}\left(\int_{\Omega_{i}}\|\sigma(h)\|^{2 p}\right)^{\frac{1}{2 p}} \geq \tilde{\lambda}_{1}(M) \int_{\Omega_{i}} \rho_{i}^{2 p} .
$$


Therefore

$$
\left(\frac{p}{\tilde{\lambda}_{1}(M)}\right)^{2 p} \int_{\Omega_{i}}\|\sigma(h)\|^{2 p} \geq \int_{\Omega_{i}} \rho_{i}^{2 p} .
$$

By $\rho_{i j} \leq \rho_{i}+\rho_{j}$ we have

$$
\left\|\rho_{i j}\right\|_{L^{2 p}\left(\Omega_{j}\right)} \leq C .
$$

The standard iteration argument in [G-T], or [Li] (for a geometric version), implies $\left\|\rho_{i j}\right\|_{L^{\infty}(K)}$ is uniformly bounded over any compact domain by some constant $C$ independent of $i$ and $j$, where $K$ is a relatively compact subset of $M$. For the completeness we sketch the argument here.

We can reduce the estimate to the $L^{\infty}$ estimate of $\rho_{i j}$ over balls. In the following, let $B_{x_{0}}(2 R) \subset \Omega_{i}$ (or briefly just $B(2 R)$ ) be a ball of fixed radius $2 R$ centered at a fixed point $x_{0}$. For the simplicity, we denote $\rho_{i j}$ by $f$, which satisfies that

$$
\int_{B(2 R)} f^{2 p} \leq C(M)
$$

for some constant $C$. By Corollary $3.5 f$ also satisfies

$$
\bar{\Delta} f \geq 0 .
$$

We will show that there exists a constant $C_{1}$ such that

$$
\sup _{B\left(\frac{R}{2}\right)} f \leq C_{1}\|f\|_{L^{2 p}} .
$$

Here the constant $C_{1}$ only depends on the geometry of $M$ and is independent of $f$. (This is a variant of the standard mean-value type inequality. One can consult Theorem 8.17 of [G-T], chapter 11 of [Li] or [L-S] for more detailed argument.)

The major geometric-analytic property of $M$ which we are going to use is the Sobolev inequality on $B(R)$. Namely, there exists a constant $C_{s}$ only dependent on the geometry of $M$ around $B(R)$ such that for any $\phi \in C_{c}^{\infty}(B(R))$

$$
\int_{B(R)}|\nabla \phi|^{2} \geq C_{s}\left(\int_{B(R)} \phi^{\frac{2 m}{m-2}}\right)^{\frac{m-2}{m}}
$$

always holds.

Before we begin the Moser iteration scheme we choose a cut-off function $\varphi$ to be

$$
\varphi(x)= \begin{cases}1 & \text { on } x \in B_{p}(d) \\ \frac{d+\sigma-r}{\sigma} & \text { on } x \in B(d+\sigma) \backslash B(d) \\ 0 & \text { on } x \in B(R) \backslash B(d+\sigma) .\end{cases}
$$


Let $a \geq p>1$. Multiplying $f^{2 a-1} \varphi^{2}$ on both sides of (4.8) and integrating by parts we get

$$
\begin{aligned}
-(2 a-1) \int_{B(R)}|\nabla f|^{2} f^{2 a-2} \varphi^{2}-2 \int_{B(R)}<\nabla f, \nabla \varphi>f^{2 a-1} \varphi & \\
& +\int_{B(R)}\langle V, \nabla f\rangle f^{2 a-1} \varphi^{2} \geq 0 .
\end{aligned}
$$

Multiplying $a$ on both sides and using Cauchy inequality we can write

$$
\begin{aligned}
& \frac{1}{\epsilon} \frac{a}{2 a-1} \int_{B(R)}\|V\|^{2} f^{2 a} \varphi^{2} \\
& -2 a \int_{B(R)}\langle\nabla f, \nabla \varphi\rangle f^{2 a-1} \varphi \geq(1-\epsilon)\left(2 a^{2}-a\right) \int_{B(R)}|\nabla f|^{2} f^{2 a-2} \varphi^{2}
\end{aligned}
$$

for any $\epsilon>0$.

On the other hand, letting $\phi=f^{a} \varphi$ we can write the following by direct calculation

$$
\begin{gathered}
a^{2} \int_{B(R)}|\nabla f|^{2} f^{2 a-2} \varphi^{2} \\
+2 a \int_{B(R)}\langle\nabla f, \nabla \varphi\rangle f^{2 a-1} \varphi+\int_{B(R)} f^{2 a}|\nabla \varphi|^{2}=\int_{B(R)}\left|\nabla\left(f^{a} \varphi\right)\right|^{2} .
\end{gathered}
$$

Choosing $1-\epsilon=\frac{a}{2 a-1}$ and adding the above two inequalities we have

$$
\frac{a}{a-1} \int_{B(R)}\|V\|^{2} f^{2 a} \varphi^{2}+\int_{B(R)} f^{2 a}|\nabla \varphi|^{2} \geq \int_{B(R)}\left|\nabla\left(f^{a} \varphi\right)\right|^{2} .
$$

Applying (4.9) to $\phi=f^{a} \varphi$ we finally have

$$
C(M, V, p)\left(1+\frac{1}{\sigma^{2}}\right) \int_{B(d+\sigma)} f^{2 a} \geq\left(\int_{B(d)} f^{a\left(\frac{2 m}{m-2}\right)}\right)^{\frac{m-2}{m}} .
$$

If we let $\nu=\frac{m}{m-2}$ the above inequality can be rewritten as

$$
\|f\|_{L^{2 a \nu}(B(d))} \leq C^{\frac{1}{a}}\left(1+\frac{1}{\sigma^{2}}\right)^{\frac{1}{a}}\|f\|_{L^{2 a}(B(d+\sigma))} .
$$

Let us now choose sequence of $a_{i}, d_{i}$, and $\sigma_{i}$ such that

$$
\begin{gathered}
a_{0}=p, a_{1}=p \nu, \cdots, a_{i}=p \nu^{i}, \cdots, \\
\sigma_{0}=\frac{1}{4} R, \sigma_{1}=2^{-1} \sigma_{0}, \cdots, \sigma_{i}=2^{-i} \sigma_{0}, \cdots,
\end{gathered}
$$


$d_{-1}=R, d_{0}=R-\sigma_{0}, d_{1}=R-\sigma_{0}-\sigma_{1}, \cdots, d_{i}=R-\sum_{j=0}^{i} \sigma_{j}, \cdots$.

Observe that $\lim _{i \rightarrow \infty} d_{i}=\frac{R}{2}$. The Moser-iteration argument completes the proof of our claim after we iterate (4.10). If we treat the usual harmonic maps we can apply Li-Schoen's mean value inequality directly since then $\rho_{i j}$ is a subharmonic function. The argument above needs $p>1$, but a general argument in [Li] shows that $p>0$ case follows from $p>1$. As a conclusion we have mean-value type inequality for any $p>0$.

Proof of theorem 4.1. In order to prove the theorem we only need to get a uniform bound for $e\left(u_{i}\right)$. By Lemma 3.1

$$
\tilde{\Delta} e\left(u_{i}\right) \geq-C e\left(u_{i}\right)
$$

for some constant $C$ only dependent on $M$ and $N$. Therefore

$$
\Delta e\left(u_{i}\right)+\left\langle V, \nabla e\left(u_{i}\right)\right\rangle \geq-C e\left(u_{i}\right) .
$$

One can easily check that the similar argument in the last paragraph can show that the mean-value type inequality also holds here for $e\left(u_{i}\right)$. Therefore we can reduce the point-wise estimate of $e\left(u_{i}\right)$ to an integral estimate of $e\left(u_{i}\right)$. This implies that in order to get the uniform estimate of $e\left(u_{i}\right)$ over the ball $B_{o}(R)$ we only need to estimate the integral of them over $B_{o}(r)$ for $r>2 R$.

Let $G(x, y)$ be the positive Green's function on $B_{o}(2 r)$ satisfying the Dirichlet boundary condition, and let $\varphi$ be a cut-off function supported in $B_{o}(2 r)$ with $\varphi=1$ in $B_{o}(r)$. Then we have

$$
\rho_{i j}^{2}(o)=-\int_{B_{o}(2 r)} G(x, y) \Delta\left(\varphi \rho_{i j}^{2}\right) .
$$

Using Lemma 3.4 and the fact that $u_{i}, u_{j}$ are Hermitian harmonic, we have

$$
\tilde{\Delta} \rho_{i j}^{2} \geq 2 \int_{0}^{1}\left\|\nabla d U\left(\frac{\partial}{\partial s}\right)\right\|^{2} d s .
$$

Hence we can write

$$
\begin{aligned}
\Delta\left(\rho_{i j}^{2} \varphi\right)= & \left(\Delta \rho_{i j}^{2}\right) \varphi+2\left\langle\nabla \rho_{i j}^{2}, \nabla \varphi\right\rangle+(\Delta \varphi) \rho_{i j}^{2} \\
= & \left(\tilde{\Delta} \rho_{i j}^{2}\right) \varphi+\left\langle V, \nabla \rho_{i j}^{2}\right\rangle+2\left\langle\nabla \rho_{i j}^{2}, \nabla \varphi\right\rangle+(\Delta \varphi) \rho_{i j}^{2} \\
\geq & \left(2 \int_{0}^{1}\left\|\nabla d U\left(\frac{\partial}{\partial s}\right)\right\|^{2} d s\right) \varphi \\
& -\|V\|\left|\nabla \rho_{i j}^{2}\right| \varphi-2\left|\nabla \varphi \| \nabla \rho_{i j}^{2}\right|+(\Delta \varphi) \rho_{i j}^{2}
\end{aligned}
$$


Applying (3.8) we have

$$
\begin{aligned}
-\|V\|\left|\nabla \rho_{i j}^{2}\right| \varphi & \geq-2\|V\| \rho_{i j}\left(\int_{0}^{1}\left\|\nabla d U\left(\frac{\partial}{\partial s}\right)\right\|^{2} d s\right)^{\frac{1}{2}} \varphi \\
& \geq-\frac{1}{2}\left(\int_{0}^{1}\left\|\nabla d U\left(\frac{\partial}{\partial s}\right)\right\|^{2} d s\right) \varphi-2\|V\|^{2} \rho_{i j}^{2} \varphi
\end{aligned}
$$

and

$$
\begin{aligned}
-2|\nabla \varphi|\left|\nabla \rho_{i j}^{2}\right| & \geq-4|\nabla \varphi| \rho_{i j}\left(\int_{0}^{1}\left\|\nabla d U\left(\frac{\partial}{\partial s}\right)\right\|^{2} d s\right)^{\frac{1}{2}} \\
& \geq-\frac{1}{2}\left(\int_{0}^{1}\left\|\nabla d U\left(\frac{\partial}{\partial s}\right)\right\|^{2} d s\right) \varphi-8 \frac{|\nabla \varphi|^{2}}{\varphi} \rho_{i j}^{2} .
\end{aligned}
$$

Therefore

$$
\begin{aligned}
\Delta\left(\rho_{i j}^{2} \varphi\right) \geq & \left(\int_{0}^{1}\left\|\nabla d U\left(\frac{\partial}{\partial s}\right)\right\|^{2} d s\right) \varphi \\
& -\left(2\|V\|^{2}-8 \frac{|\nabla \varphi|^{2}}{\varphi}-\Delta \varphi\right) \rho_{i j}^{2} \\
\geq & \frac{1}{2}\left(e\left(u_{i}\right)-3 e\left(u_{j}\right)\right) \varphi-\left(2\|V\|^{2}-8 \frac{|\nabla \varphi|^{2}}{\varphi}-\Delta \varphi\right) \rho_{i j}^{2} .
\end{aligned}
$$

In the last step we have used Proposition 3.2. Now we have

$$
\begin{aligned}
\rho_{i j}^{2}(o)= & -\int_{B_{o}(2 r)} G(x, y) \Delta\left(\varphi \rho_{i j}^{2}\right) \\
\leq & -\frac{1}{2}\left(\int_{B_{o}(2 r)} G(x, y) \varphi\left(e\left(u_{i}\right)-3 e\left(u_{j}\right)\right) d s\right) \\
& +C \int_{B_{o}(2 r)} G(x, y) \rho_{i j}^{2} .
\end{aligned}
$$

Fixing $j$ and using the fact $\rho_{i j}$ is uniformly bounded we can get

$$
\int_{B_{o}(r)} e\left(u_{i}\right) G(x, y) \leq C .
$$

Here $C$ is independent of $i$. From the above inequality and the fact that $G(x, y)$ is positive on $B_{o}(r)$ we can easily see

$$
\int_{B_{o}(r)} e\left(u_{i}\right) \leq C .
$$


Hence we have $e\left(u_{i}\right) \leq C$ for some constant $C$ which is independent of $i$. Therefore the $\left\{u_{i}\right\}$ has a subsequence which converges to $u$, a solution of (1.1). Thus we complete the proof of Theorem 4.1.

We should point out here that by our construction we can see easily that $\rho(u(x), h(x))$, the homotopy distance between the initial map $h$ and $u$, belongs to $L^{2 p}(M)$. We will show in the next section that under this condition the solution is also unique if we assume that the difference vector fields $V$ has bounded $L^{\infty}$ norm.

\section{Uniqueness}

In this section we will show that the Hermitian harmonic map we constructed in last section is in fact unique if we assume that the vector fields $V$ satisfies $\|V\|_{L^{\infty}}<\infty$

Theorem 5.1. Assume that $M$ has positive first eigenvalue $\tilde{\lambda}_{1}(M)$ for $\tilde{\Delta}$, $N$ has nonpositive sectional curvature. Let $u_{1}$ and $u_{2}$ be two homotopy Hermitian harmonic maps from $M$ to $N$, and let $\rho(x)$ be the homotopy distance between them. If there is a positive number $p \geq 1$ such that $\rho(x) \in$ $L^{2 p}(M)$ and the difference vector fields $V$ satisfies $\|V\|_{L^{\infty}}<\infty$, then $u_{1}(x)=u_{2}(x)$.

Proof. By the remark after the proof of Theorem 4.1 we know that $\rho(u(x)$, $h(x)) \in L^{2 p}(M)$ if $u$ is the solution of (1.1) from our construction. Therefore if $u_{1}$ and $u_{2}$ are two solutions from our construction we have that $\rho\left(u_{1}(x), u_{2}(x)\right) \leq \rho\left(u_{1}(x), h(x)\right)+\rho\left(u_{2}(x), h(x)\right)$ and belongs to $L^{2 p}(M)$ also. Because of that Theorem 5.1 gives the uniqueness result corresponding to Theorem 4.1.

The proof follows closely from the $L^{p}$ Liouville type theorem of Yau (See [Li], Sect. 7). By Corollary 3.5 we have

$$
\tilde{\Delta} \rho \geq 0 \text {. }
$$

Let $\varphi$ be a cut-off function. Multiply $\rho^{2 p-1} \varphi^{2}$ on both sides of the above inequality and integrate by parts. Then

$$
\begin{gathered}
-(2 p-1) \int_{M}|\nabla \rho|^{2} \rho^{2 p-2} \varphi^{2}-2 \int_{M}<\nabla \rho, \nabla \varphi>\rho^{2 p-1} \varphi \\
+\int_{M}\langle V, \nabla \rho\rangle \rho^{2 p-1} \varphi^{2} \geq 0 .
\end{gathered}
$$

Letting $\phi=\rho^{p} \varphi$ and using the assumption that

$$
\int_{M}(-\tilde{\Delta} \phi) \phi \geq \tilde{\lambda}_{1}(M) \int_{M} \phi^{2}
$$


We can write the following inequality

$$
\begin{aligned}
p^{2} \int_{M} \rho^{2 p-2}|\nabla \rho|^{2} \varphi^{2} & +2 p \int_{M}\langle\nabla \rho, \nabla \varphi\rangle \rho^{2 p-1} \varphi+\int_{M} \rho^{2 p}|\nabla \varphi|^{2} \\
& -p \int_{M}\langle, V, \nabla \rho\rangle \rho^{2 p-1} \varphi^{2}-\int_{M}\langle V, \nabla \varphi\rangle \rho^{2 p} \varphi \\
& \geq \tilde{\lambda}_{1}(M) \int_{M} \rho^{2 p} \varphi^{2}
\end{aligned}
$$

Multiplying (5.1) by $p$ and adding (5.1) to the above inequality, then we have

$$
\begin{aligned}
\int_{M} \rho^{2 p}|\nabla \varphi|^{2}-\int_{M}\langle V, \nabla \varphi\rangle \rho^{2 p} \varphi \geq & \tilde{\lambda}_{1}(M) \int_{M} \rho^{2 p} \varphi^{2} \\
& +\left(p^{2}-p\right) \int_{M} \rho^{2 p-2}|\nabla \rho|^{2} \varphi^{2},
\end{aligned}
$$

which implies

$$
\begin{aligned}
\frac{1}{r^{2}} \int_{B(2 r)} \rho^{2 p}+\frac{1}{r}\|V\|_{L^{\infty}} \int_{B(2 r)} \rho^{2 p} \geq & \int_{M} \rho^{2 p}|\nabla \varphi|^{2}-\int_{M}\langle V, \nabla \varphi\rangle \rho^{2 p} \varphi \\
\geq & \tilde{\lambda}_{1}(M) \int_{B(r)} \rho^{2 p} \\
& +\left(p^{2}-p\right) \int_{B(r)} \rho^{2 p-2}|\nabla \rho|^{2} .
\end{aligned}
$$

Letting $r \rightarrow \infty$ we have that the left hand side of the above inequality goes to 0 . Hence

$$
\int_{M} \rho^{2 p} \leq 0
$$

which implies the conclusion of Theorem 5.1.

\section{Global existence for the heat equation}

In this section we will treat the heat equation (1.2). We are going to show that the heat equation (1.2) has a global solution under the assumption that $\tilde{\lambda}_{1}(M) \geq 0$, which is weaker than the assumption of Theorem 4.1. Also we show that the solution of the heat equation will converges to a Hermitian harmonic map if $\tilde{\lambda}_{1}(M)>0$ and $\|V\|_{L^{\infty}}<\infty$. For existence it does not tell more than Theorem 4.1 but it provides the homotopy explicitly by the heat flow and coincides with the uniqueness result of Sect. 5. 
Theorem 6.1. Let $M$ be a complete Hermitian manifold with nonnegative $\tilde{\lambda}_{1}(M) \geq 0$, and let $N$ be a complete Riemannian manifold with nonpositive sectional curvature. Let $h$ be a smooth map with bounded $\|\sigma(h)\|_{2 p}, p \geq 1$. Then there exists a solution $u(x, t): M \times[0, \infty) \rightarrow N$, which solves (1.2) and is homotopic to $h$ with $u(x, 0)=h(x)$.

Proof. Here we use the compact exhaustion argument as before. Let $\Omega_{i}$ be a sequence of exhaustion domain of $M$. From [J-Y] we know that the following Dirichlet problems have solutions $u_{i}(x, t)$ on $\Omega_{i} \times[0, \infty)$;

$$
\left\{\begin{array}{l}
\frac{\partial u_{i}}{\partial t}=\sigma\left(u_{i}\right) \\
u(x, 0)=h(x), \\
\left.u_{i}(x, t)\right|_{\partial \Omega_{i}}=h(x), u_{i} \sim h \text { rel } \partial \Omega_{i} .
\end{array}\right.
$$

We will show that there exists a subsequence converging to a solution $u(x, t)$ by establishing uniform gradient estimates for every $K \times[0, T]$, where $K$ is a compact subdomain of $M$.

First we are going to show that $\left\|\frac{\partial u_{i}}{\partial t}\right\|$ are uniformly bounded on $K \times$ $[0, T]$. From $[\mathrm{J}-\mathrm{Y}]$ we know that $\left\|\frac{\partial u_{i}}{\partial t}\right\|=\left\|\sigma\left(u_{i}\right)\right\|$ satisfies

$$
\left\{\begin{array}{l}
\left(\tilde{\Delta}-\frac{\partial}{\partial t}\right)\left\|\frac{\partial u_{i}}{\partial t}\right\| \geq 0 \\
\left.\left\|\frac{\partial u_{i}}{\partial t}\right\|\right|_{\Omega_{i}}=0
\end{array}\right.
$$

In fact it was shown in [J-Y] (See the paragraph before Lemma 1 of [J-Y]) that

$$
\left(\tilde{\Delta}-\frac{\partial}{\partial t}\right)\left\|\frac{\partial u_{i}}{\partial t}\right\|^{2} \geq 2\left\|\nabla d u_{i}\left(\frac{\partial}{\partial t}\right)\right\|^{2}
$$

which is weaker than what we need. Since it is easy to check that

$$
\left|\nabla\left(\left\|\frac{\partial u_{i}}{\partial t}\right\|\right)\right|^{2} \leq\left\|\nabla d u_{i}\left(\frac{\partial}{\partial t}\right)\right\|^{2}
$$

One can get (6.2) from the above two inequalities easily.

It is not hard to check that the Moser-iteration argument of Sect. 4 applies to parabolic equations also. Therefore we can reduce the point-wise estimates to the $L^{2 p}$ estimates, which can be done as follows. 
For the simplicity of the notation we denote $\left\|\frac{\partial u_{i}}{\partial t}\right\|(x, t)$ by $g(x, t)$. Direct calculation shows that

$$
\begin{aligned}
\frac{\partial}{\partial t} \int_{\Omega_{i}} g^{2 p}= & 2 p \int_{\Omega_{i}} g^{2 p-1} g_{t} \\
\leq & 2 p \int_{\Omega_{i}} g^{2 p-1} \tilde{\Delta} g \\
= & 2 p \int_{\Omega_{i}} g^{2 p-1}(\Delta g+\langle V, \nabla g\rangle) \\
= & -2 p(2 p-1) \int_{\Omega_{i}}|\nabla g|^{2} g^{2 p-2}+2 p \int_{\Omega_{i}}<V, \nabla g>g^{2 p-1} \\
\leq & -2 p(2 p-1) \int_{\Omega_{i}}|\nabla g|^{2} g^{2 p-2}+2 p\|V\|_{\infty, \Omega_{i}} \int_{\Omega_{i}}|\nabla g| g^{2 p-1} \\
\leq & -2 p(2 p-1) \int_{\Omega_{i}}|\nabla g|^{2} g^{2 p-2} \\
& +2 p\|V\|_{\infty, \Omega_{i}}\left(\int_{\Omega_{i}}|\nabla g|^{2} g^{2 p-2}\right)^{\frac{1}{2}}\left(\int_{\Omega_{i}} g^{2 p}\right)^{\frac{1}{2}} \\
\leq & \frac{2 p\|V\|_{\infty, \Omega_{i}}}{2 p-1} \int_{\Omega_{i}} g^{2 p} .
\end{aligned}
$$

Hence

$$
\|g\|_{L^{2 p}\left(\Omega_{i}\right)} \leq \exp \left(\frac{T\|V\|_{\infty, \Omega_{i}}}{2 p-1}\right)\|\sigma(h)\|_{L^{2 p}(M)}
$$

for all $t \in[0, T]$. Therefore we conclude that for any compact subset $K \subset \Omega_{i}$ there exists a constant $C=C(T, M, V, K)$ such that

$$
\left\|\frac{\partial u_{i}}{\partial t}\right\|=\left\|\sigma\left(u_{i}\right)\right\| \leq C
$$

for any $(x, t) \in K \times[0, T]$. The rest is to get uniform estimates for $e\left(u_{i}\right)$. This can be done similarly to the elliptic case of Sect. 4. First we derive the estimates of $\left\|\rho_{i j}\right\|_{L^{\infty}(K \times[0 . K])}$, which rely on the estimates of $\left\|\rho_{i}\right\|_{L^{\infty}(K \times[0, K])}$. Then, using the mean value inequality, we can reduce down to the $L^{2 p}$ estimates of $\rho_{i}$. Before we start the proof we should point out here that if $u_{i}$ solves (1.2), the Hermitian harmonic map heat equation, one can have all those differential inequalities of Sect. 3 after replacing $\tilde{\Delta}$ by $\left(\tilde{\Delta}-\frac{\partial}{\partial t}\right)$. For example, $\rho_{i}$ satisfy the following

$$
\left\{\begin{array}{l}
\left(\tilde{\Delta}-\frac{\partial}{\partial t}\right) \rho_{i} \geq-\|\sigma(h)\|, \\
\rho_{i}(x, 0)=0, \\
\left.\rho_{i}\right|_{\partial \Omega_{i}}=0 .
\end{array}\right.
$$


And $\rho_{i j}$ satisfy

$$
\left\{\begin{array}{l}
\left(\tilde{\Delta}-\frac{\partial}{\partial t}\right) \rho_{i j} \geq 0 \\
\rho_{i j}(x, 0)=0 .
\end{array}\right.
$$

Multiply $\rho_{i}^{2 p-1}$ on both sides of (6.3) and integrate by parts. With the help of the boundary and initial conditions we have

$$
\begin{aligned}
& -(2 p-1) p \int_{\Omega_{i}}\left|\nabla \rho_{i}\right|^{2} \rho_{i}^{2 p-2}+p \int_{\Omega_{i}}\left\langle V, \nabla \rho_{i}\right\rangle \rho_{i}^{2 p-1}-p \int_{\Omega_{i}}\left(\rho_{i}\right)_{t} \rho_{i}^{2 p-1} \\
& =p \int_{\Omega_{i}}\left(\tilde{\Delta} \rho_{i}\right) \rho_{i}^{2 p-1}-p \int_{\Omega_{i}}\left(\rho_{i}\right)_{t} \rho_{i}^{2 p-1} \\
& \geq-p \int_{\Omega_{i}}\|\sigma(h)\| \rho_{i}^{2 p-1} .
\end{aligned}
$$

By our assumption we have that for any smooth cut-off function $\phi$,

$$
\int_{M}|\nabla \phi|^{2}-\int_{M}\langle V, \nabla \phi\rangle \geq \tilde{\lambda}_{1}(M) \int_{M} \phi^{2} .
$$

Letting $\phi=\rho_{i}^{p}$ in the above inequality we have

$$
p^{2} \int_{\Omega_{i}}\left|\nabla \rho_{i}\right|^{2} \rho_{i}^{2 p-2}-p \int_{\Omega_{i}}<V, \nabla \rho_{i}>\rho_{i}^{2 p-1} \geq \tilde{\lambda}_{1}(M) \int_{\Omega_{i}} \rho_{i}^{2 p} .
$$

Adding this to the inequality after (6.4) we have

$$
\begin{aligned}
p\left(\int_{\Omega_{i}}\|\sigma(h)\|^{2 p}\right)^{\frac{1}{2 p}}\left(\int_{\Omega_{i}} \rho_{i}^{2 p}\right)^{\frac{2 p-1}{2 p}} & \geq p \int_{\Omega_{i}}\|\sigma(h)\| \rho_{i}^{2 p-1} \\
& -\left(p^{2}-p\right) \int_{\Omega_{i}}\left|\nabla \rho_{i}\right|^{2} \rho_{i}^{2 p-2} \\
& \geq p \int_{\Omega_{i}}\left(\rho_{i}\right)_{t} \rho_{i}^{2 p-1}+\tilde{\lambda}_{1}(M) \int_{\Omega_{i}} \rho_{i}^{2 p} \\
& =\frac{1}{2} \frac{\partial}{\partial t}\left(\int_{\Omega_{i}} \rho_{i}^{2 p}\right)+\tilde{\lambda}_{1}(M) \int_{\Omega_{i}} \rho_{i}^{2 p} \\
& \geq \frac{1}{2} \frac{\partial}{\partial t}\left(\int_{\Omega_{i}} \rho_{i}^{2 p}\right) .
\end{aligned}
$$

Together with the initial condition that $\rho_{i}(x, 0)=0$ the above differential inequality implies that

$$
\left\|\rho_{i}\right\|_{L^{2 p}} \leq\left((4 p-1)\|\sigma(h)\|_{\left.L^{2 p} T\right)^{\frac{1}{4 p-1}}} .\right.
$$


Because of that $\rho_{i j} \leq \rho_{i}+\rho_{j}$, (6.4) and the mean-value type inequality(which can be proved by a Moser-iteration argument as in the Sect. 4) there exists a constant $C=C(V, M, K, T)$ such that

$$
\left\|\rho_{i j}\right\|_{L^{\infty}(K \times[0, T])} \leq C
$$

After we establish the $L^{\infty}$ estimates for $\rho_{i j}$ we can finally derive the uniform estimates for $e\left(u_{i}\right)$. Just as in the elliptic case the key point here again relies on a differential type inequality. First from $[\mathrm{J}-\mathrm{Y}]$ we have the following inequality for the energy density function $e\left(u_{i}\right)$

$$
\left(\tilde{\Delta}-\frac{\partial}{\partial t}\right) e\left(u_{i}\right) \geq-C e\left(u_{i}\right), \text { for some } C \text { independent of } u_{i},
$$

by which we can reduce the $L^{\infty}$ estimates to the integral estimates as in the elliptic case. The key point here is that the differential inequality (6.5) implies a mean-value type inequality.

Furthermore we have the similar inequality as Lemma 3.4 for $\rho_{i j}^{2}$ as follows

$$
\left(\tilde{\Delta}-\frac{\partial}{\partial t}\right) \rho_{i j}^{2} \geq 2 \int_{0}^{1}\left\|\nabla d U\left(\frac{\partial}{\partial s}\right)\right\|^{2} d s
$$

This is a parabolic version of Lemma 3.4. Here we also have used the fact that $u_{i}$ and $u_{j}$ solve the parabolic Hermitian harmonic map equation (1.2).

Now we can derive the integral estimates for $e\left(u_{i}\right)$ as what we did in the Sect. 4. By the reasoning above it will be sufficient to estimate the integral of $e\left(u_{i}\right)$ over $B_{o}(r) \times[0, T]$ as what we did in the Sect. 4. (Since by the parabolic mean-value inequality we know that $\sup _{B_{o}\left(\frac{r}{2}\right) \times[0, T]} e\left(u_{i}\right)$ is bounded from above by the sum of the average of $e\left(u_{i}\right)$ over $B_{o}(r) \times[0, T]$ and $2 \sup _{B_{o}(2 r)} e(h)$. One can consult Theorem 1.4 of [L-T] for more details on the mean-value type inequality for parabolic equations.)

Let $H(x, y, t)$ be the Dirichlet heat kernel on $B_{o}(2 r)$, and let $\varphi$ be a cut-off function supported in $B_{o}(2 r)$ and $\varphi=1$ in $B_{o}(r)$. Then, using the fact that $\rho_{i j}(x, 0)=0$, we have

$$
\rho_{i j}^{2}(o)=-\int_{0}^{T} \int_{B_{o}(2 r)} H(x, y, t)\left(\Delta-\frac{\partial}{\partial t}\right)\left(\varphi \rho_{i j}^{2}\right) d v d t .
$$


Similar to the last part of the proof of Theorem 4.1 one can write

$$
\begin{aligned}
\left(\Delta-\frac{\partial}{\partial t}\right)\left(\rho_{i j}^{2} \varphi\right)= & \left(\left(\Delta-\frac{\partial}{\partial t}\right) \rho_{i j}^{2}\right) \varphi+2\left\langle\nabla \rho_{i j}^{2}, \nabla \varphi\right\rangle+(\Delta \varphi) \rho_{i j}^{2} \\
= & \left(\left(\tilde{\Delta}-\frac{\partial}{\partial t}\right) \rho_{i j}^{2}\right) \varphi+\left\langle V, \nabla \rho_{i j}^{2}\right\rangle+2\left\langle\nabla \rho_{i j}^{2}, \nabla \varphi\right\rangle \\
& +(\Delta \varphi) \rho_{i j}^{2} \\
\geq & \left(2 \int_{0}^{1}\left\|\nabla d U\left(\frac{\partial}{\partial s}\right)\right\| d s\right) \varphi-\|V\|\left|\nabla \rho_{i j}^{2}\right| \\
& -2\left|\nabla \varphi \| \nabla \rho_{i j}^{2}\right|+(\Delta \varphi) \rho_{i j}^{2} .
\end{aligned}
$$

In the last inequality we have used inequality (6.4). By the same calculation as in the last part of the proof of Theorem 4.1 we can have

$$
\begin{aligned}
\Delta\left(\rho_{i j}^{2} \varphi\right) \geq & \left(\int_{0}^{1}\left\|\nabla d U\left(\frac{\partial}{\partial s}\right)\right\|^{2} d s\right) \varphi \\
& -\left(-2\|V\|^{2}-8 \frac{|\nabla \varphi|^{2}}{\varphi}-\Delta \varphi\right) \rho_{i j}^{2} \\
\geq & \frac{1}{2}\left(e\left(u_{i}\right)-3 e\left(u_{j}\right)\right) \varphi-\left(-2\|V\|^{2}-8 \frac{|\nabla \varphi|^{2}}{\varphi}-\Delta \varphi\right) \rho_{i j}^{2} .
\end{aligned}
$$

Plug the above inequality into (6.7). Then we have

$$
\begin{aligned}
\rho_{i j}^{2}(o)= & -\int_{0}^{T} \int_{B_{o}(2 r)} H(x, y, t)\left(\Delta-\frac{\partial}{\partial t}\right)\left(\varphi \rho_{i j}^{2}\right) d v d t \\
\leq & -\frac{1}{2} \int_{0}^{T} \int_{B_{o}(2 r)} H(x, y, t) \varphi\left(e\left(u_{i}\right)-3 e\left(u_{j}\right)\right) d v d t \\
& +C \int_{0}^{T} \int_{B_{o}(2 r)} H(x, y, t) \rho_{i j}^{2} d v d t .
\end{aligned}
$$

Fixing $j$ and using the fact that $\rho_{i j}$ is uniformly bounded we have

$$
\int_{0}^{T} \int_{B_{o}(r)} H(x, y, t) e\left(u_{i}\right) d v d t \leq C .
$$

Therefore

$$
\int_{0}^{T} \int_{B_{o}(2 r)} e\left(u_{i}\right) d v d t \leq C,
$$

for some constant $C$ independent of $i$. Then we complete the proof of Theorem 6.1 . 
Remark 6.1. From the proof we can see that we did not use a lot of our assumption $\tilde{\lambda}_{1}(M) \geq 0$. By the knowledge about the usual harmonic maps we believe that the existence of the heat equation can be proved under much more relaxed conditions. Since we won't use it for our purpose of constructing Hermitian harmonic maps we leave it to the interested reader.

Corollary 6.2. Assume that $M$ has positive first eigenvalue $\tilde{\lambda}_{1}(M)$ for $\tilde{\Delta}$, $N$ has nonpositive sectional curvature and $h$ is a smooth map from $M$ to $N$. Furthermore we assume $\|V\|_{L^{\infty}}<\infty$. If there is a positive number $p \geq 1$ such that the initial map $h$ has bounded $\|\sigma(u)\|_{L^{2 p}}(p \geq 1)$ then there exists a solution $u(x, t)$ for the Hermitian harmonic map heat equation such that $u(x, 0)=h(x)$ and $u(x, t)$ converges to a Hermitian harmonic map as $t \rightarrow \infty$.

Proof. From Theorem 6.1 we know that there exists a global solution $u(x, t)$ for the Hermitian harmonic heat equation (1.2). The only thing we need to show here is the convergence of the $u(x, t)$ as $t \rightarrow \infty$. It will be sufficient to prove the result of Corollary 6.2 if we can show that $\left\|\frac{\partial u}{\partial t}\right\| \rightarrow 0$ as $t \rightarrow \infty$. Recall that from [J-Y] (Or see the proof of (6.2)) we have

$$
\left\{\begin{array}{c}
\left(\tilde{\Delta}-\frac{\partial}{\partial t}\right)\left\|\frac{\partial u}{\partial t}\right\| \geq 0, \\
\left\|\frac{\partial u}{\partial t}\right\|(x, 0)=\|\sigma(h)\| .
\end{array}\right.
$$

Let $f(x, t)=\left\|\frac{\partial u}{\partial t}\right\|$. Since

$$
\begin{aligned}
\tilde{\Delta} f^{\alpha} & =\alpha f^{\alpha-1} \Delta f+\alpha(\alpha-1) f^{\alpha-2}|\nabla f|^{2}+\alpha f^{\alpha-1}<V, \nabla f> \\
& =\alpha f^{\alpha-1} \tilde{\Delta} f+\alpha(\alpha-1) f^{\alpha-2}|\nabla f|^{2}
\end{aligned}
$$

and

$$
\frac{\partial}{\partial t} f^{\alpha}=\alpha f^{\alpha-1} f_{t}
$$

we have that

$$
\left\{\begin{array}{l}
\left(\tilde{\Delta}-\frac{\partial}{\partial t}\right) f^{\alpha} \geq 0, \\
f^{\alpha}(x, 0)=\|\sigma(h)\|^{\alpha}
\end{array}\right.
$$

Hence we have

$$
\begin{aligned}
\left(\left\|\frac{\partial u}{\partial t}\right\|(x, t)\right)^{\alpha} & =f^{\alpha}(x, t) \\
& \leq \int_{M} \bar{H}(x, y, t)\|\sigma(h)\|^{\alpha} d y \\
& \leq\left(\int_{M} \bar{H}^{q^{\prime}}(x, y, t) d y\right)^{\frac{1}{q^{\prime}}}\left(\int_{M}\|\sigma(h)\|^{\alpha p^{\prime}}\right)^{\frac{1}{p^{\prime}}} .
\end{aligned}
$$


Here $\bar{H}(x, y, t)$ is the heat-kernel of $\tilde{\Delta}$ and $\frac{1}{q^{\prime}}+\frac{1}{p^{\prime}}=1$. We choose $\alpha$ and $p^{\prime}$ such that $\alpha p^{\prime}=2 p$ and $\alpha$ close to $2 p \geq 2$ such that $p^{\prime}$ is close to 1 . Therefore the proof depends on the $L^{q^{\prime}}$ estimate of the heat-kernel $\bar{H}(x, y, t)$ of $\tilde{\Delta}$. By the choice of $p^{\prime}$ we can assume that $q^{\prime}>2$. The similar estimate for the Laplacian-Beltrami heat-kernel was derived in [L-T] and [Gr]. For the completeness we include a proof here. (It has been modified a little for our case.)

Let $\varphi$ be a cut-off function on $M$ and $2 q=q^{\prime}$. Direct calculation gives

$$
\begin{aligned}
\frac{d}{d t}\left(\int_{M} \bar{H}^{2 q}(x, y, t) \varphi^{2} d y\right)= & 2 q \int_{M} \bar{H}^{2 q-1} \bar{H}_{t} \varphi^{2} d y \\
= & 2 q \int_{M} \bar{H}^{2 q-1}(\tilde{\Delta} \bar{H}) \varphi^{2} d y \\
= & -2 q(2 q-1) \int_{M} \bar{H}^{2 q-2}|\nabla \bar{H}|^{2} \varphi^{2} \\
& +2 q \int_{M} \bar{H}^{2 q-1}\langle V, \nabla \bar{H}\rangle \varphi^{2} \\
& -4 q \int_{M} \bar{H}^{2 q-1}\langle\nabla \bar{H}, \nabla \varphi\rangle \varphi .
\end{aligned}
$$

By the assumption on $\tilde{\lambda}_{1}(M)$ with $\phi=\bar{H}^{q} \varphi$ we have that

$$
\begin{aligned}
& 2 q^{2} \int_{M} \bar{H}^{2 q-2}|\nabla \bar{H}|^{2} \varphi^{2}+4 q \int_{M} \bar{H}^{2 q-1} \varphi\langle\nabla \bar{H}, \nabla \varphi\rangle \\
& +2 \int_{M} \bar{H}^{2 q}|\nabla \varphi|^{2}-2 q \int_{M} \bar{H}^{2 q-1} \varphi^{2}\langle\nabla \bar{H}, V\rangle-2 \int_{M} \bar{H}^{2 q}\langle\nabla \varphi, V\rangle \varphi \\
& \quad=2\left(\int_{M}|\nabla \phi|^{2}-\int_{M}\langle V, \nabla \phi\rangle\right) \\
& \quad \geq 2 \tilde{\lambda}_{1}(M) \int_{M} \phi^{2} \\
& \quad=2 \tilde{\lambda}_{1}(M) \int_{M} \bar{H}^{2 q} \varphi^{2} .
\end{aligned}
$$

Adding the above two inequalities we have

$$
\begin{aligned}
\frac{d}{d t}\left(\int_{M} \bar{H}^{2 q}(x, y, t) \varphi^{2} d y\right)+2 \tilde{\lambda}_{1}(M)\left(\int_{M} \bar{H}^{2 q}(x, y, t) \varphi^{2} d y\right) \\
\leq-\left(2 q^{2}-2 q\right) \int_{M} \bar{H}^{2 q-2}|\nabla \bar{H}|^{2} \varphi^{2} \\
\quad+2 \int_{M} \bar{H}^{2 q}|\nabla \varphi|^{2}-2 \int_{M} \bar{H}^{2 q}\langle\nabla \varphi, V\rangle \varphi \\
\leq 2 \int_{M} \bar{H}^{2 q}|\nabla \varphi|^{2}-2\|V\|_{L^{\infty}} \int_{M} \bar{H}^{2 q}|\nabla \varphi| \varphi
\end{aligned}
$$


Letting $r \rightarrow \infty$ we have that

(6.10) $\frac{d}{d t}\left(\int_{M} \bar{H}^{2 q}(x, y, t) d y\right)+2 \tilde{\lambda}_{1}(M)\left(\int_{M} \bar{H}^{2 q}(x, y, t) d y\right) \leq 0$.

Therefore we have

$$
\left(\int_{M} \bar{H}^{2 q}(x, y, t) d y\right) \leq \exp \left(-2 \tilde{\lambda}_{1}(M)(t-1)\right)\left(\int_{M} \bar{H}^{2 q}(x, y, 1) d y\right) .
$$

Now we complete our proof of Corollary 6.2.

Remark 6.2. From the proof one can see that the above argument also works for $1 \geq p>\frac{1}{2}$ if one assume the existence of the heat equation. Even though we need the assumption $\|V\|_{L^{\infty}}<\infty$ to prove the convergence to a Hermitian harmonic map this assumption coincides with the assumption for the uniqueness.

\section{References}

[Ch] Cheng, S.Y.: Liouville theorems for harmonic maps, Proc. Symp. Pure Math. 36 (1980), 147-151

[D] Ding, W-Y.: Harmonic maps of complete noncompact Riemannian manifolds, International J. Math. 2 (1991), 617-633

[Gr] A. Grigor'yan.: Heat kernal upper bounds on a complete non-compact manifold, Rev. Math. Iberoamericana. 10 (1994), 395-452

[G-T] Gilbarg, D., Trudinger,N.S.: Elliptic partial differential equations of second order, 2nd edition, Springer-Verlag, 1983

[J-Y] Jost, J., Yau, S.T.: A nonlinear elliptic system and rigidity theorems, Acta Math. 170 (1993), 221-254

[Li] Li, P.: Lecture notes on geometric analysis, Research Institute of Mathematics, Global Analysis Center Seoul National University, 1993

[LJ] Li, J.: The heat flows and harmonic maps of complete noncompact Riemannian manifolds, Math. Z. 198 (1993), 161-173

[L-T] Li, P., Tam, L.F.: The heat equation and harmonic maps of complete manifolds, Invent. Math. 105 (1991), 1-46

[L-W] Li, P., Wang, J.: Convex hull properties of harmonic maps, J. Differential Geometry 48 (1998), 457-530

[S] Schoen, R.: Analytic aspect of the harmonic map problem, MSRI Publ. 2 (1984), 321-358

[S-Y] Schoen, R., Yau, S.T: On univalent harmonic maps between surfaces, Invent. Math. 44 (1978), 265-278 